found any other reports of pneumomediastinum as a complication of anorexia nervosa, starvation, or wasting diseases. Studies on rats of lung ultrastructure and mechanics after prolonged starvation have shown an increase in alveolar surface forces and a decrease in tissue elasticity, with fewer lamellar bodies in pulmonary granular pneumocytes (the storage site for surfactant) and a decrease in volume density of mitochondria in the same cells. ${ }^{3}$ Perhaps comparable changes occur in the human lung after starvation and account for this complication in anorexia nervosa.

We thank Dr J R Rees, United Bristol hospitals, and Dr A D Perrett, Royal Cornwall Hospital, for permission to report these cases under their care.

${ }^{1}$ Feighner, J P, et al, Archives of General Psychiatry, 1972, 26, 57.

${ }^{2}$ Al-Mufty, N S, and Bevan, D H, British Fournal of Clinical Practice, 1977, 31, 160.

${ }^{3}$ Sahebjami, H, Vassallo, C L, and Wirman, J A, American Review of Respiratory Disease, 1978, 117, 77

(Accepted 12 September 1978)

Department of Medicine, British Royal Infirmary, Bristol BS2 8HW ANITA J DONLEY, MB, MRCP, senior house officer (present address: Southmead Hospital, Bristol)

Royal Cornwall Hospital, Treliske, Truro, Cornwall $\mathrm{T}$ J KEMPLE, MB, DCH, senior house officer in medicine

\section{Strokes and hypertension: contribution of poor blood pressure control}

There is a well-established relation between the incidence of strokes and the adequacy of blood pressure control in hypertension. ${ }^{1}$ There is, however, little information available on the reasons for poor control in hypertensive patients who have a stroke, ${ }^{2}$ and we have investigated the problem further.

\section{Patients, methods, and results}

All patients under the age of 75 who were admitted to hospital over eight months with a completed stroke (signs persisting for more than 24 hours) were seen by one of us (PK). Whether or not the patient was hypertensive was assessed on the basis of clinical history and examination, chest $x$-ray and electrocardiograph, reference to previous hospital notes, and interviews with relatives. General practitioners who did not know the aims of the investigation were asked by letter about previous blood pressure readings and treatment. The response rate was $88 \%$. Relatives were also asked about how regularly the patient took his drugs. A diastolic blood pressure of $110 \mathrm{~mm} \mathrm{Hg}$ or more was the criterion used for hypertension, and in most of the previously diagnosed cases several readings were available. Patients were considered to have had good blood pressure control if a diastolic blood pressure of $100 \mathrm{~mm} \mathrm{Hg}$ or less had been recorded within the previous six months, and a history was obtained of regular medication until the stroke.

Of the 65 patients investigated, $35(27 \mathrm{men})$ proved to be hypertensive (age 47-74, median 66), and only three of these were not previously known to have hypertension. Twelve $(38 \%)$ of the 32 known hypertensive patients were believed to have had well-controlled blood pressure before the stroke. No fewer than 11 patients had discontinued hypotensive medication on the advice of their doctor (seven patients) or spontaneously (four patients) up to six years beforehand and in five cases within two weeks to a month before the stroke. Two other patients admitted to taking their drugs only intermittently, and three patients had never had treatment. Four patients had had unsatisfactory blood pressure control in spite of taking regular medication.

\section{Comment}

The assessment of previous blood pressure control is difficult even in patients regularly attending hypertension clinics, which only four of our patients were doing. Our figure of $62 \%$ for the incidence of unsatisfactory blood pressure control in patients who suffer hypertensive strokes is, however, close to the figure of Beevers et al of $70 \% .1$ In a review of the "obstacles to controlling hypertension" Stason $e t a l^{3}$ found that failure to start treatment and to maintain it were the most important. The clinician must also accept some responsibility for the non-compliance of patients to prescribed medication. The need for and the importance of life-long treatment is often not emphasised enough, unnecessarily complicated drug regimens are prescribed and too little attention is paid to side effects.

Our study was started after one of the patients at our hypertension clinic suffered his second stroke because of non-compliance and poor blood pressure control; we had failed to recognise that this was due to impotence from methyldopa. Pharmacological resistance was also noted, but it appears to be less important than the other reasons for poor control.

In our study nearly one-sixth of the hypertensive strokes occurred within one month of stopping treatment. A relation between stroke and stopping hypotensive therapy has a theoretical basis in loss of autoregulation of cerebral blood flow with fairly acute short-term rises in blood pressure. ${ }^{4}$ This suggests that our observation may be important clinically. Nevertheless, it seems unreasonable to think that the potential danger of stopping hypotensive treatment should discourage the initiation of treatment when otherwise indicated.

Only three of our patients presented with completed strokes and undiagnosed hypertension. These findings provide evidence that more effort needs to be paid to ensuring adequate treatment of patients already known to have hypertension than to finding new cases. In every hypertensive patient who suffers a stroke the previous management of the hypertension should be reviewed. In this way local obstacles to controlling hypertension can be identified and possibly overcome and the incidence of stroke reduced. The means of ensuring adequate treatment need to be widely publicised. ${ }^{5}$

We thank our colleagues in hospital and general practice for their helpful collaboration

${ }^{1}$ Beevers, D G, et al, Postgraduate Medical fournal, 1973, 49, 905.

2 World Health Organisation, Hypertension and Stroke Control in the Community. Geneva, WHO, 1976.

${ }^{3}$ Stason, W B, Weinstein, M C, and Shepard, D S, in Hypertension: $A$ Policy Perspective, ed M C Weinstein and W B Stason, p 111. Cambridge, Mass, and London. Harvard University Press, 1976.

${ }^{4}$ Russell, R R W, Lancet, 1975, 2, 1283.

${ }^{5}$ Stamler, R, et al, Lancet, 1975, 2, 1227.

(Accepted 27 September 1978)

Whittington Hospital, London N19

P G E KENNEDY, MB, MRCP, medical registrar

B I HOFFBRAND, DM, FRCP, consultant physician

\section{Cardiac pain referred to site of previously experienced somatic pain}

Cardiac $\mathrm{p}^{- \text {in }}$ may be referred at times to the site of pain from lesions in the heau and neck. For example, the pain of angina may be felt in the same distribution as pain from cervical spondylosis or from the teeth (for instance, a periapical abscess). ${ }^{12}$ More rarely, cardiac pain may be atypically referred to the site of pain previously experienced in the chest or upper limbs. We describe three such cases.

\section{Case roorts}

(1) A 56-year-old man, a clerk, presented with asymptomatic essential hypertension in 1960 and was treated with methyldopa. In 1965 he complained of back - in at the level of $\mathrm{T} 4$ which was strictly related to exertion and was relieveci oy rest and by trinitrin. He had no precordial pain. An ECG showed infe-ior ischaemic changes. In 1968 he had an inferior myocardial infarction, but even then pain was confined to the dorsal region over T4. A radiograph showed an old compression fracture of $T 4$. On direct questioning he said he had injured his back in falling from a horse 20 years previously. The patient died suddenly after his discharge home. There was no necropsy.

(2) A 56-year-old solicitor had had left thoracic herpes zoster 30 months previously. He had mild postherpetic neuralgia, but was otherwise well. In September 1975 his neuralgic pain suddenly worsened and was accompanied by sweating and nausea but no precordial pain. Because the pain was similar, though more severe, he thought it was due to an exacerbation of his herpes zoster. He attended the emergency department, where he was found 
to have a regular pulse with ectopic beats, a fourth sound, and coarse basa crackles. There was a post-herpetic scar extending laterally on the left side of the chest in the T4 dermatome. The ECG showed a typical anterior myocardial infarction, and there were confirmatory enzyme changes. Recovery was uneventful.

(3) A bricklayer aged 60 had a head injury in 1960. This was followed by pain in both arms. In 1968 a neurologist found no abnormal physical signs but $x$-ray examination showed a disc lesion at C4-5. He presented in 1975 with pain radiating to both arms in the distribution of his previous chronic pain but with no chest pain. He was admitted, despite the atypical history, because of ECG changes diagnostic of anteroseptal myocardial infarction. These evolved as usual and were accompanied by enzyme changes. He made an uneventful recovery.

\section{Comment}

These three cases share the characteristic that their cardiac pain, when it occurred, was at the site of previous undoubted somatic pain. The term "facilitation" has been used to describe this type of radiation of cardiac pain, ${ }^{3}$ implying that pain impulses reaching the thoracic cord followed pathways previously established by a somatic injury and suggesting some form of somatic memory for pain. It is now known that somatic input can suppress visceral input in the cells of the dorsal horn of the cord, and that interactions of inputs can be modified by impulses descending from the brain. ${ }^{4}$ The precise mechanism by which previous somatic pain leads to altered localisation of visceral pain remains unclear, though it may be mediated at a cortical level. We were unable to try to suppress the pain in our cases with local anaesthesia as reported in cases of cardiac pain referred to a phantom limb. ${ }^{5}$ This, however, is a different phenomenon, where the pain follows a "normal" distribution. Referred cardiac pain should be borne in mind in a patient with unusual chest pain and the possibility of other local disease considered in patients with cardiac pain of atypical distribution.

${ }^{1}$ Cohen, H, Transactions of the Medical Society of London, 1944, 64, 65.

2 Oram, S, Clinical Heart Disease, 1st edn. London, Heinemann, 1971.

${ }^{3}$ Mackenzie, J, Brain, 1893, 16, 321.

${ }^{4}$ Pomeranz, B, Wall, P D, and Weber, W V, Journal of Physiology, 1968, 199, 511 .

${ }^{5}$ Cohen, H, and Jones, H W, British Heart fournal, 1943, 5, 67.

(Accepted 28 September 1978)

\section{Whittington Hospital, London N19 5NF}

J A HENRY, MB, MRCP, medical registrar (present address: Department of Clinical Pharmacology, St Bartholomew's Hospital, London EC1A 7BE)

E MONTUSCHI, MD, FRCP, consulting physician

\section{An outpatient medication record}

In view of the increasing use of potent medicines an accurate record of all medicines is essential for adequate patient care. We report here the introduction of a medication record sheet to outpatient clinics in the Aberdeen teaching hospitals.

\section{Methods and results}

The number of outpatient attendances arising from each new referral, the number of medicines recorded at each attendance, and the number of different specialist clinics attended concurrently were considered when designing the sheet. It was designed to facilitate recording information about all medicines, whether advised by general practitioners, recommended by hospital doctors, taken as self-medication, or administered in the outpatient clinics. The sheet (see figure) was used on a trial basis in five different specialist outpatient clinics. The doctors were asked to record all medicines taken by patients in the two weeks before the patient's first attendance at the clinic and all medicines they recommended. They were asked to update the record at subsequent visits so that information about current medication was prominently displayed. After four months of general use the amount of information recorded and the adequacy of the format of the record were assessed by examining the case notes of patients subsequently attending the different clinics. On the day before the appointment the case notes were scrutinised and details about medication were obtained from the most recent hospital and general practitioner letters. Entries made in the medication record sheet were also noted. On the day the patients attended they were interviewed ( $\mathrm{ZGE}, \mathrm{TAJ}$ ) about details of current medication, including self medication, after their consultation with the doctor. The notes of these patients were subsequently re-examined, and information about medicines in the medication record sheet was compared with the information obtained from correspondence and at the interview. We repeated this procedure for patients returning for further review.

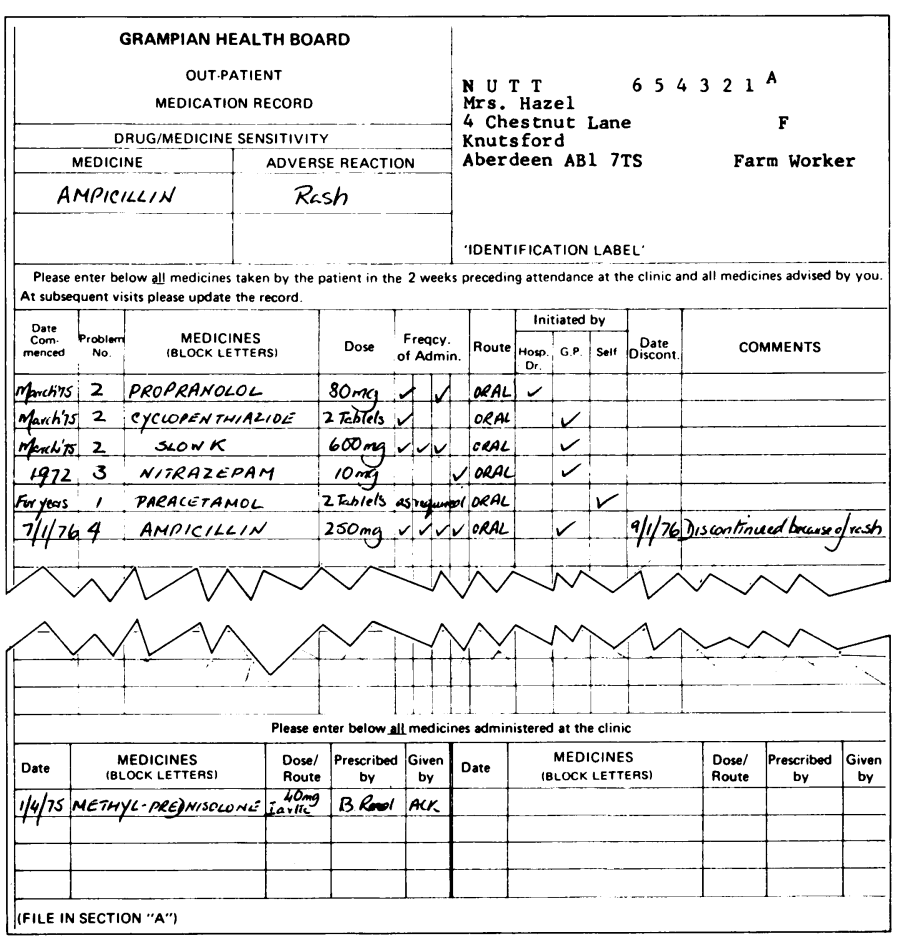

Example of partially completed outpatient medication record.

In the run-in period 1027 patients attended the five clinics. Of these only 162 patients were eligible for study because an outpatient attendance was planned within the subsequent six months. Sixteen patients failed to attend. No information appeared on the sheet for a further 48 patients, although medicines had been either prescribed or recommended for 26 of these. O the remaining 98 patients (average age 55 years; $51 \mathrm{men}$ ), 22 had empty record sheets, and they confirmed at interview that they had not been taking any medication. For the remaining 76, 333 medicines were ascertained either at interview, from the correspondence, or from the medication record. Altogether $270(81 \%)$ medicines were recorded on the medication record sheet; fewer than $1 \%$ had been taken as self-medication. Of the remainder (63 medicines, 44 patients) not recorded on the medication record sheet, 15 $(5 \%)$ had been taken as self-medication, the commonest being analgesics, while the $48(15 \%)$ prescribed medicines comprised mainly analgesics, hypnotics, and oral contraceptives. The case notes of the 44 patients for whom 63 medicines had not been recorded on first attendance were reexamined. All attended for further review, and two-thirds ( 40 prescriptions) of the omissions were subsequently recorded. By the second consultation $93 \%$ of the medicines were recorded on the outpatient medication sheet. Al columns in the sheet except the optional "date commenced," "problem number," and "comments" were completed in at least $90 \%$ of cases. During the trial the average number of lines used on a first visit was 3.3.

\section{Comment}

Standardised hospital drug recording procedures such as that introduced in Aberdeen by Crooks et $a l^{1}$ have proved of value, and, because of failures in communication about medicines taken by patients attending outpatient departments, ${ }^{2}$ an outpatient sheet was developed along similar principles.

The trial showed that the format of the sheet was acceptable in five different clinics. It has now been accepted for general use, and further studies are planned to ascertain its usefulness. Although the recording required some effort, the results during the study showed that the recording procedure was fairly satisfactory, providing information not previously available in a readily identifiable form in the case notes. The procedure will, however, always be deficient in certain respects and some compromise is necessary between the aim of recording total information about drugs and the willingness of doctors to record. Even with this limitation, use of the sheet should improve the transfer of information about drugs between doctors and patients, increasing the 